\title{
Remote supratentorial hemorrhage after posterior fossa surgery: A brief case report
}

\author{
Luis Rafael Moscote-Salazar ${ }^{1}$, Gabriel Alcalá-Cerra ${ }^{1}$, Hernando \\ Raphael Alvis-Miranda², Omar Ramírez ${ }^{3}$, Willem Guillermo \\ Calderón-Miranda ${ }^{4}$, Andrés M. Rubiano ${ }^{5}$
}

${ }^{1}$ Neurosurgeon, Universidad de Cartagena, Colombia

${ }^{2}$ Physician, Universidad de Cartagena, Colombia

${ }^{3}$ Neurosurgeon, Universidad El Bosque, Colombia

${ }^{4}$ Physician, Universidad del Magdalena, Colombia

${ }^{5}$ Neurosurgeon, Hospital Universitario de Neiva, Colombia

\begin{abstract}
The supratentorial hemorrhage after posterior fossa surgery is an unusual but delicate complication that carries high mortality and morbidity. A 50 year old woman presented vertigo 6 months of evolution, which worsened in the last 2 months accompanied by ataxia. She showed left cerebellar signs, had no focal motor or sensory deficits. A brain MRI identified cerebellopontine angle lesion with mass effect. The patient was treated on suboccipital craniectomy and resection of right posterior fossa tumor, the histopathological diagnosis was consistent with typical meningioma. (WHO Class I).

The postoperative period was satisfactory. A month later, presented clinical symptoms of right-sided hemiparesis, brain CT revealed left frontal supratentorial hematoma, receiving conservative management. Patient was
\end{abstract}

discharged after 10 days. Reports in the literature on this rare complication, detailed cases where the hematoma was presented in hours to days. To our knowledge this is the first report in the literature of supratentorial hemorrhage and posterior fossa surgery one month after the surgical procedure has been performed.

Key words: Meningioma, posterior fossa surgery, supratentorial hemorrhage, Neurosurgery

\section{Introduction}

Remote supratentorial hematoma after posterior fossa surgery for the removal of a space-occupying lesion is a rare but dramatic and dreaded complication, carrying significant morbidity and mortality. We describe a rare complication of extensive supratentorial hemorrhage following posterior fossa surgery; review the relevant 
literature and discus the possible cause of hemorrhage in the present case.

\section{Case report}

A 50-year-old woman had a history of headache and progressive ataxia over 1 year. Neurological examination showed a horizontal nystagmus, a slight gait ataxia, right dysmetria and bilaterally papilledema. CT scan also revealed a right meningioma of $6 \times 5 \times 5,5 \mathrm{~cm}$ in size in the right posterior fossa with moderate hydrocephalus (Figure 1). Preoperative right carotid and right vertebral artery angiographic scan has been done. The patient underwent suboccipital craniectomy in sitting position and ventricular drainage has been performed to prevent air embolism. So intraoperative course was uneventful and tumor removed totally [Figure - 2]. In early postoperative neurological examination was normal but after six hours she became somnolent and developed a hemiplegia on the left site.

A new CT scan revealed a hyperdense lesion of $2 \times 1,5 \times 4 \mathrm{~cm}$ in size in the right frontoparietal region [Figure - 3]. The patient was treated conservatively and the patient's neurologic state improved in five days. CT scan revealed a hypodense lesion in the right fronto-parietal region in subsequent second month.

\section{Discusion}

Supratentorial intracerebral hemorrhage is uncommon after posterior fossa surgery.
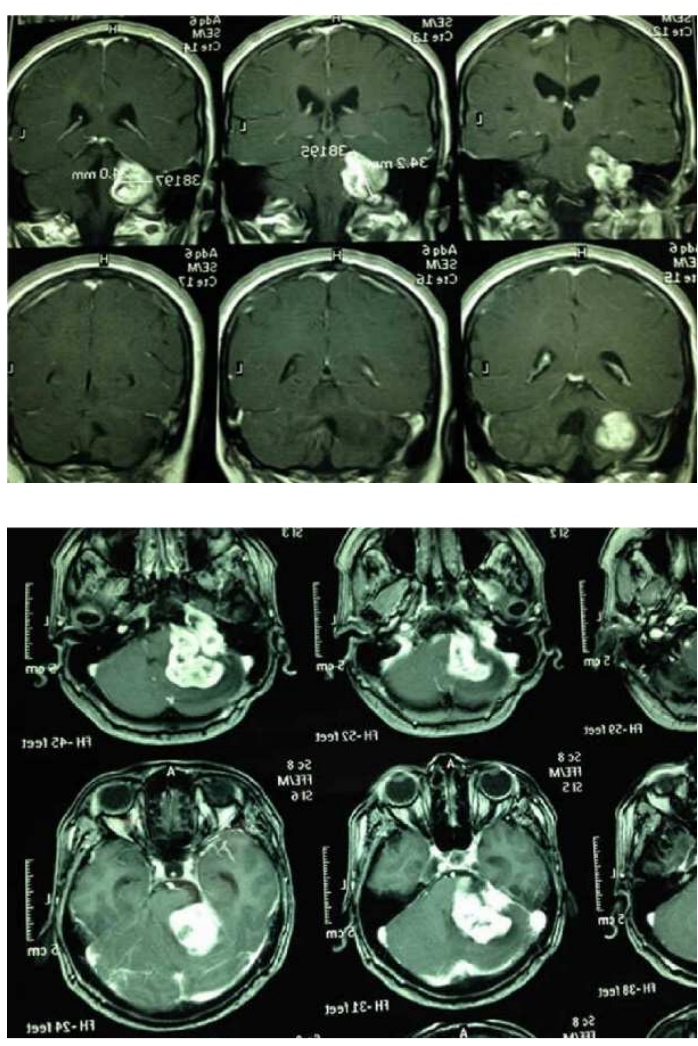

Figures 1 and 2 - Preoperative brain T1W MRI contrast images showing large posterior fossa tumor on right side with mass effect

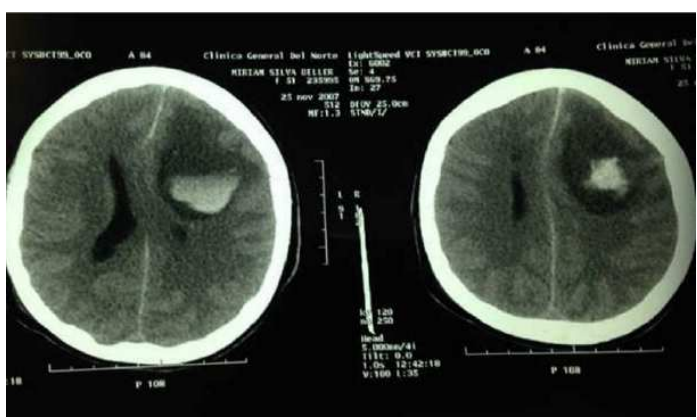

Figure 3 - Postoperative CT scan revealed a hyperdense lesion of $2 \times 1,5 \times 4$

The mechanism for producing the bleeding is not entirely certain, but it is proposed that in the case where the sitting position is used, a 
DOI: 10.2478/romneu-2014-0049

decrease of cerebral blood flow and then cerebral ischemia occurs. By switching the position after surgery hyperperfusion areas leads to bleeding in ischemic areas. Heines et al reported 5 supratentorial hemorrhages after posterior fossa surgery in 825 patients firstly. The patients had neither coagulopathy nor predisposant factors. [1,2]

TABLE 1

Summary of published supratentorial intracerebral hemorrhage after posterior fossa surgery

\begin{tabular}{|c|c|c|c|c|c|}
\hline Author and year & Age/Gender & Diagnosis & Localization & POP interval & Sequela \\
\hline Haines, 1978 & $\begin{array}{l}65 / \mathrm{F} \\
55 / \mathrm{F} \\
41 / \mathrm{F} \\
64 / \mathrm{F} \\
62 / \mathrm{F}\end{array}$ & $\begin{array}{l}\text { Neuralgia V right } \\
\text { Neuralgia IX right } \\
\text { Neuralgia V right } \\
\text { An. painful right } \\
\text { Schwanoma VIII } \\
\text { left }\end{array}$ & $\begin{array}{l}\text { Right Occipital } \\
\text { Ringht basal ganglia } \\
\text { Right Frontoparietal } \\
\text { Left frontal } \\
\text { Intraventricular }\end{array}$ & $\begin{array}{l}4 \mathrm{hrs} \\
45 \mathrm{~min} \\
\text { inmediate } \\
18 \text { horas } \\
\text { Inmediate }\end{array}$ & -- \\
\hline Standefer, 1984 & $59 /--$ & Meningioma & Basal ganglia & 7 days & -- \\
\hline Harders, 1985 & $\begin{array}{l}44 / \mathrm{F} \\
51 / \mathrm{F} \\
58 / \mathrm{M}\end{array}$ & $\begin{array}{l}\text { Meningioma } \\
\text { Schwnoma VIII } \\
\text { Meningioma }\end{array}$ & $\begin{array}{l}\text { Left frontoparietal } \\
\text { Bilateral frontal } \\
\text { Left parietal }\end{array}$ & $\begin{array}{l}-- \\
-- \\
--\end{array}$ & -- \\
\hline Seiler, 1986 & $\begin{array}{l}66 / \mathrm{F} \\
64 / \mathrm{F} \\
59 / \mathrm{F}\end{array}$ & $\begin{array}{l}\text { Schwnoma VIII } \\
\text { Right } \\
\text { Right meningioma } \\
\text { Right Schwnoma }\end{array}$ & $\begin{array}{l}\text { Right Parietal } \\
\text { Left parietal } \\
\text { Right occipital }\end{array}$ & $\begin{array}{l}\text { Inmediate } \\
3 \mathrm{hrs} \\
24 \mathrm{hrs}\end{array}$ & -- \\
\hline Kalfas, 1988 & -- & -- & -- & -- & -- \\
\hline Bucciero, 1991 & $46 / \mathrm{F}$ & Hemangioblastoma & $\begin{array}{l}\text { Left } \\
\text { Temporoparietal }\end{array}$ & $6 \mathrm{hrs}$ & -- \\
\hline Prieto, 1993 & $45 / F$ & $\begin{array}{l}\text { Left } \\
\text { VIII }\end{array}$ & Left Frontoparietal & $16 \mathrm{hrs}$ & -- \\
\hline Tondon, 2004 & $\begin{array}{l}30 / \mathrm{F} \\
50 / \mathrm{F}\end{array}$ & $\begin{array}{l}\text { schwannomas } \\
\text { Astrocitoma }\end{array}$ & $\begin{array}{l}\text { Left } \\
\text { Parietaloccipital } \\
\text { Basal ganglia }\end{array}$ & $\begin{array}{l}24 \mathrm{hrs} \\
6 \mathrm{hrs}\end{array}$ & $\begin{array}{l}\text { Died } \\
\text { Died }\end{array}$ \\
\hline Kalkan, 2006 & $63 / \mathrm{F}$ & Meningioma & -- & $6 \mathrm{hrs}$ & -- \\
\hline Agrawal, 2010 & $47 / F$ & --- & Right Temporal & $3 \mathrm{hrs}$ & -- \\
\hline Moscote, 2014 & $50 / \mathrm{F}$ & Meningioma & Left frontal & 30 days & $\begin{array}{l}\text { Right } \\
\text { hemiparesis }\end{array}$ \\
\hline
\end{tabular}

F: female M: male

Our patient underwent tumor resection in the benck park position. No ventricular drain was placed. In the preoperative and postoperative periods coagulation parameters were normal. The values of blood pressure preoperatively, intraoperatively and postoperatively were normal. Bleeding was probably caused by the rupture of perforating 
veins of the basal ganglia or in the subependymal region.

\section{Conclusion}

The supratentorial hemorrhage after posterior fossa surgery is an unusual but delicate complication that carries high mortality and morbidity. Reports in the literature on this rare complication, evidence cases where the hematoma was presented in hours to days. $(3,4,5,6,7,8,9,10)$. To our knowledge this is the first report in the literature of supratentorial hemorrhage and posterior fossa surgery one month after the surgical procedure has been performed.

\section{Correspondence}

Dr. Luis Rafael Moscote-Salazar, Universidad de Cartagena, Cartagena de Indias, Colombia, e-mail: mineurocirujano@aol.com

\section{References}

1. Tondon A, Mahapatra AK. Superatentorial intracerebral hemorrhage following infratentorial surgery. J Clin Neurosci. 2004;11:762-5. [PubMed]
2. Haines SJ, Maroon JC, Jannetta PJ. Supratentorial intracerebral hemorrhage following posterior fossa surgery. J Neurosurg. 1978;49:881-6. [PubMed]

3.Kalkan E, Eser O. Supratentorial intracerebral haemorrhage following posterior fossa operation. Neurol India. 2006;54:220-1.

4. Bucciero A, Quaglietta P, Vizioli L. Supratentorial intracerebral hemorrhage after posterior fossa surgery: Case report. J Neurosurg Sci. 1991;35:221-4. [PubMed] 5. Harders A, Gilsbach J, Weigel K. Supratentorial space occupying lesions following infratentorial surgery early diagnosis and treatment. Acta Neurochir (Wien) 1985;74:57-60. [PubMed]

6. Vrettou CS, Stavrinou LC, Halikias S, Kyriakopoulou M, Kollias S, Stranjalis G, et al. Factor XIII deficiency as a potential cause of supratentorial haemorrhage after posterior fossa surgery. Acta Neurochir (Wien) 2010;152:529-32. [PubMed]

7. Wolfsberger S, Gruber A, Czech T. Multiple supratentorial epidural haematomas after posterior fossa surgery. Neurosurg Rev. 2004;27:128-32. [PubMed]

8.Pandey P, Madhugiri VS, Sattur MG, Devi BI. Remote supratentorial extradural hematoma following posterior fossa surgery. Childs Nerv Syst. 2008;24:851-4. [PubMed] 9. Seiler RW, Zurbrugg HR. Supratentorial intracerebral hemorrhage after posterior fossa operation. Neurosurgery. 1986;18:472-4. [PubMed]

10. Agrawal A, Kakani A, Ray K. Extensive Supratentorial Hemorrhages Following Posterior Fossa Meningioma Surgery. J Surg Tech Case Rep. 2010 JulDec; 2(2): 87-89. 